Sharif University of Technology
Scientia Iranica
Transactions E: Industrial Engineering
hCIENTIA $/ /$ scientiairanica.sharif.edu
IRANICA

\title{
The business advantage of identifying and solving pseudo-continuous-integer periodical linear problems
}

\author{
F. Trigos ${ }^{\mathrm{a}}$ and L.E. Cárdenas-Barrón ${ }^{\mathrm{b}, *}$ \\ a. Tecnologico de Monterrey, EGADE Business School, Ave. Eugenio Garza Lagüeray Rufino Tamayo, San Pedro Garza García, \\ N.L., México, 66269. \\ b. Tecnologico de Monterrey, School of Engineering and Sciences, Ave. Eugenio Garza Sada 2501, Monterrey, N.L., México, 64849.
}

Received 28 November 2019; received in revised form 31 July 2020; accepted 26 October 2020

\author{
KEYWORDS \\ Business profit; \\ Integer programming; \\ Linear programming; \\ Operations \\ management; \\ Operations planning.
}

\begin{abstract}
Many optimization applications require the final value of decision variables to be integer. In many cases, the relaxed optimal solution does not satisfy the integrality constraint; therefore, the problem must be solved using integer or mix-integer programming algorithms with significant computational effort and most likely a worsen objective function value. The contribution of this paper is two-fold: (a) identification of a type of problems in which the relaxed optimal objective function value can be kept at the implementation phase by modifying the planning horizon and (b) identification of a multi-period-based solution procedure. Three small instances are provided in order to illustrate the methodology as well as the economic impact involved. In addition, a fourth industrial-scale case is included for the benefit of practitioners. This work shows that business profit can be increased for pseudo-continuous-integer periodical linear problems by identifying optimal decisionmaking periods.

(C) 2022 Sharif University of Technology. All rights reserved.
\end{abstract}

\section{Introduction}

In a competitive business environment, practitioners are interested in solutions that can be obtained and implemented in a simple way. They require that the generated solutions provide better profits (or lower costs) in order to promote business growth and competitiveness.

Linear, integer, and mixed integer programming models are a flourishing field of optimization. Nowadays, they are applied to an immense variety of reallife problems in a number of disciplines [1-10]. The development of these models has provided the ground for enhancement of efficient solutions over decades and

*. Corresponding author. Tel.: +52 8112089477 E-mail addresses: ftrigos@tec.mx (F. Trigos); lecarden@tec.mx (L.E. Cárdenas-Barrón)

doi: $10.24200 /$ sci.2020.54580.3815 they are still progressing quickly. For linear models, the well-known simplex method derived in [11], the interior point method developed in [12], and the one later improved in [13] are used to solve this class of problems. For integer programming models, algorithms and methods such as branch and bound [14], cutting planes [15], and branch and cut [16] exist. It is important to remark that the integer and mixed integer programming models are complex problems, many of which are NP-hard. Therefore, the computational complexity is high with often long computational time.

The process of obtaining the solution of integer and mix integer models is far more complex than that of simple linear programming ones. A particular network problem called transshipment enjoys unique characteristics such that if all demands are integer, all vertexes in the feasible region are integers; therefore, the solution of the network simplex $[11,17]$ is integer without considering the problem as an integer model.

Often, there is a loss in the objective function 
value when a problem is transformed from continuous to integer (or mix-integer). Hence, the search to reduce this loss is a continuous concern for practitioners and academics. There is scant treatment in the operational research academic literature in this regard; therefore, this concern has become the main motivation of this paper.

The contribution of this article is two-fold. The first is identifying a special class of problems whose solution must be integer while the continuous solution is not. However, an integer solution can be obtained by identifying special characteristics of the application. The second contribution is providing a method to convert the continuous solution into an integer solution (which can be implemented) without losing value in the objective function for these kinds of problems.

This paper is organized as follows: Section 2 presents a class of problems (to be named pseudocontinuous-integer periodical linear problems) in which its special characteristics are the focus of this work. Section 3 includes the methodology to transform a continuous solution from this class of problems into an integer one. Section 4 presents three small numerical instances to illustrate the functionality of the method. An industrial size case is represented for the benefit of practitioners. Finally, Section 5 provides conclusions and further research.

\section{Special characteristics of pseudo-continuous integer periodical linear problems}

Let us define a Pseudo-Continuous-Integer Periodical Linear Problem (PCIPLP) as the one that satisfies the following five characteristics:

1. Pure integer programming problems with no binary variables;

2. Single period planning horizon that repeats identically over a non-limited number of consecutive periods;

3. The time to make decisions can be transformed from every period to once every $T$ periods, where $T$ is an integer number to be determined;

4. Between two consecutive periods, fractional values (resources, demands, etc.) can be conveyed;

5. The objective function of the problem can change from a fixed periodical number to an average per period.

Many special applications in practice satisfy the above conditions including service management (public and private), transportation, production, order acceptance [18] and manufacturing, among others. Some illustrations are included in Section 4.

\section{Methodology}

Let us consider an integer problem that meets the characteristics of the latter section. Let the integer period problem be:

$$
\begin{aligned}
& \text { Opt } \quad c^{t} x, \\
& \text { s.t.: } \\
& A x=b, \quad x \in \mathbb{Z}^{n+},
\end{aligned}
$$

where $A \in \mathbb{R}^{m \times n}, c \in \mathbb{R}^{n}, b \in \mathbb{R}^{m}, x \in \mathbb{Z}^{n+}$, and $\mathbb{Z}^{n+}$ be the $n$ dimensional setof non-negative integers.

The relaxed linear model associated to Eq. (1) is:

$$
\begin{aligned}
& \text { Opt } \quad c^{t} y, \\
& \text { s.t.: } \\
& \text { Ay }=b, \quad y \geq 0,
\end{aligned}
$$

where $y \in \mathbb{R}^{n}$. Let $I=\{1, \cdots, n\}$ be the index set and $y^{*}$ be the optimal solution of Eq. (2), with $y_{i}^{*}=d_{i} / e_{i}$, where $d_{i}, e_{i} \in \mathbb{Z}^{+} \forall i \in I$. It is important to remark that all optimal linear programming solutions involve rational numbers because of the computational nature of the algorithms [19]. Let $T$ be the minimum common multiple of all $e_{i}$ 's.

By multiplying both sides of the constraint $A y=$ $b$ by the scalar $T$ in Eq. (2) and making $w_{i}=T y_{i}$, $\forall i \in I$. Then, the one period model in Eq. (2) is transformed into a $T$-period model as follows:

$$
\text { Opt } \quad c^{t} w
$$

s.t.

$$
\begin{aligned}
& A w=T b, \\
& w \geq 0,
\end{aligned}
$$

where $w \in \mathbb{R}^{n}$. Relation (3) represents the objective function value taking into account $T$ periods and Eq. (4) represents the technical constraints for a $T$ period problem.

Notice that the model in Eq. (2) is mathematically equivalent to Relations (3) to (5); however, the latter happens to have integer solutions for all its variables. Since both problems share the same $A, c$, and $T>$ 0 , they share the same optimal basis (basic columns of matrix $A$ ) and the value for the dual variables. Thus, the sensitivity linear programming tools apply to Relations (3) to (5). Hence, sensitivity analysis interpretations are valid on Relations (3) to (5) as long as the resulting solution remains with all integer values. The latter can be a challenging task.

Besides, Problems (3) to (5) have only a change in the time horizon in Eq. (1) as long as the latter satisfies all the characteristics listed in Section 2. 
Table 1. Method for solving the single period problem in Eq. (1) through the multiple-period problem in Relations (3) through (5).

Step 1: Solve to optimality the continuous linear programming model in Eq. (2) as:

Opt $c^{t} y$

s.t. $A y=b, \quad y \geq 0$

Step 2: Let the optimal solution of Eq. (2) be $y_{i}^{*}=$ $d_{i} / e_{i}, \forall i \in I$, where $d_{i}, e_{i} \in \mathbb{Z}^{+}$

Step 3: Determine $T$ as the minimum common multiple for all $e_{i}, \forall i \in I$,

where $T$ determines the planning horizon

Step 4: Define the optimal solution of the problem in Relations (3) through (5) as:

Opt $c^{t} w$

s.t.: $A w=T b, \quad w \geq 0$

as $w_{i}^{*}=\left(\frac{T}{d_{i}}\right) e_{i}$, where $w_{i}^{*} \in \mathbb{Z}^{+}, \forall i \in I$.

Step 5: Sensitivity analysis, since the problem in Relations (3) through (5) is a continuous linear programming problem with optimal solution $w_{i}^{*} \in \mathbb{Z}^{+}$, all regular sensitivity analysis techniques are available for solving the problem in Relations (3) through (5) as long as the new solution remains integer

As a matter of fact, all the constraints in Eq. (4) from $A y=b$ in Eq. (2) have just moved their limits parallel-wise by a factor $T$.

The optimal solution of Relations (3) to (5) is given by:

$$
w_{i}^{*}=\left(\frac{T}{e_{i}}\right) d_{i}, \quad \forall i \in I,
$$

where $\frac{T}{e_{i}} \in \mathbb{Z}^{+}$; thus, $w_{i}^{*} \in \mathbb{Z}^{+}$is an optimal solution to Relations (3) to (5).

Notice that the problem in Relations (3) to (5) is a regular linear programming problem that occurs to have integer solutions for all its variables. Hence, all sensitivity analysis techniques are applied as long as the new solution remains integer.

In summary, a problem that satisfies the conditions of a PCIPLP in Section 2 is re-formulated from a single period to a $T$ period planning horizon with an optimal solution as in Eq. (6) by solving the linear programming continuous problem in Eq. (2).

The pseudo-code in Table 1 transforms the planning horizon for decision-making from one period to $T$ periods for PCIPLPs.

\section{Numerical illustrations}

Four PCIPLP cases are presented in this section. Three of these cases are small instances to illustrate the methodology in simple terms, while the last one represents an industrial size order acceptance case to show that the methodology can be applied to larger problems.

\subsection{A public service management instance}

A small city of 15,000 inhabitants consumes an average of 1,200,000 liters of drinkable water per day. The city obtains water from the central purifying facility where water is treated by conventional filtration and chloro-hydration methods. In addition, two chemical compounds (softener and purifier) are included. The city is evaluating two potential suppliers of these chemical compounds. Supplier $A$ offers packages with $4 \mathrm{~kg}$ of softener and $1.5 \mathrm{~kg}$ of purifier for $\$ 80$ a package. Supplier $B$ offers packages with $2 \mathrm{~kg}$ of softener and $4.5 \mathrm{~kg}$ of purifier for $\$ 100$ a package. In order to keep water drinkable, the city facility requires $75 \mathrm{~kg}$ of softener and $50 \mathrm{~kg}$ of purifier per day. The objective is to provide the daily levels of softener and purifier at a minimum cost for the city [20].

Let $x_{A}$ and $x_{B}$ be the number of packages per day to buy from each supplier. The relaxed linear programming model for the daily decision is:

$$
\begin{array}{lll}
\min & 80 y_{A}+100 y_{B}, & \\
\text { s.t.: } & & \\
\text { Softener: } & 4 y_{A}+2 y_{B} & \geq 75, \\
\text { Purifier: } & 1.5 y_{A}+4.5 y_{B} & \geq 50, \\
& y_{A}, y_{B} & \geq 0,
\end{array}
$$

with optimal continuous solution $y_{A}=95 / 6$ and $y_{B}=$ $35 / 6$ and optimal daily cost of $\$ 1,850.00$.

The city cannot buy fractional packages from the suppliers. If the integrality constraint is added for both variables, the solution changes to $x_{A}=16$ and $x_{B}=6$ with an optimal solution of $\$ 1,880.00$. These values represent a $\$ 30.00$ increment per day, which is approximately $1.62 \%$ of increase in the daily cost.

Since the daily requirements of softener and purifier are fixed and the minimum common multiple in the denominator of the decision variables is $T=6$, the city can buy every six days 95 packages $\left(w_{A}^{*}\right)$ from supplier $A$ and 35 packages $\left(w_{B}^{*}\right)$ from supplier $B$.

Because the packages have separated containers for every element (softener and purifier), the city must measure $75 \mathrm{~kg}$ of softener and $50 \mathrm{~kg}$ of purifier every day and apply them to the city water supply. The comprehensive purchase will cost $\$ 11,100.00$ every six days; meanwhile, the average daily cost remains at its minimum at $\$ 1,850.00$.

The problem in Relation (7) is a numerical version of this problem in Eq. (2), while Problem (8) is equivalent to Relations (3) to (5): 


$$
\begin{array}{lll}
\min & 80 w_{A}+100 w_{B}, & \\
\text { s.t.: } & & \\
\text { Softener } T: & 4 w_{A}+2 w_{B} & \geq 75 \times 6=450, \\
\text { Purifier } T: & 1.5 w_{A}+4.5 w_{B} & \geq 50 \times 6=300, \\
& w_{A}, w_{B} & \geq 0 .
\end{array}
$$

Figure 1 shows the three solutions $x^{*}, y^{*}$, and $w^{*}$. One can notice that the feasible region of Relation (8) is limited by the constraints Softener $T$ and Purifier $T$ and has re-scaled (moved parallel-wise) the boundaries of both constraints by a factor of $T=6$ from the initial problem in Relation (7), limited by the original constraints softener and purifier.

Regarding sensitivity analysis, the dual variables of Relation (8) include softener $T=-14$ and purifier $T=-16$ (both integers). Thus, if one right-hand side is moved, it must be such that the new solution remains integer. Or, one can multiply both right-hand sides by multiples of $T=6$ and keep the same dual variables and an integer solution.

In addition, the dual variables of Relation (7) are Softener $=-14$ and Purifier $=-16$ with the same values than the ones in Relation (8).

\subsection{A production mix problem}

A small manufacturing facility produces two products. Three machines are used in the manufacturing process, each with 44 available hours per week. The single period is considered as a week.

Table 2 shows operational information where columns 2-4 represent the number of manufacturing hours to produce a unit of each product. The last column in the table represents the marginal contribution obtained per product.
Table 2. Manufacturing operational data for production mix problem.

\begin{tabular}{ccccc}
\hline Product & $\begin{array}{c}\text { Compression } \\
(\mathbf{h r})\end{array}$ & $\begin{array}{c}\text { Cut } \\
(\mathbf{h r})\end{array}$ & $\begin{array}{c}\text { Polish } \\
(\mathbf{h r})\end{array}$ & $\begin{array}{c}\text { Marginal } \\
\text { contribution } \\
(\$)\end{array}$ \\
\hline 1 & 0.5 & 0.49 & 0.21 & 50 \\
2 & 0.9 & 0.7 & 0.39 & 105 \\
\hline
\end{tabular}

The problem consists of finding the optimal production mix to maximize the sum of marginal contributions obtained by the production plan. In practice, it is not possible to manufacture and deliver a fraction of a unit.

Let $x_{i}$ be the number of units of product $i$ to be produced per week, where $i=1,2$. The relaxed linear programming model follows:

$$
\begin{array}{lll}
\max & 50 y_{1}+105 y_{2}, & \\
\text { s.t.: } & & \\
\text { Compression: } & 0.5 y_{1}+0.9 y_{2} & \leq 44, \\
\text { Cut: } & 0.49 y_{1}+0.7 y_{2} & \leq 44, \\
\text { Polish: } & 0.21 y_{1}+0.39 y_{2} & \leq 44, \\
& y_{1}, y_{2} & \geq 0 .
\end{array}
$$

The optimal continuous solution is $y_{1}=a_{1} / b_{1}=0$, $y_{2}=a_{2} / b_{2}=440 / 9=48+8 / 9 \approx 48.8888 \cdots$, with an objective function value of $\$ 5,133+1 / 3$. Thus, $T=9$.

If the integrality constrain is added to Relation (9), the solution transforms to $x_{1}=1, x_{2}=48$, with objective function value $\$ 5,090.00$. This integer solution translates to $\$ 43.33$ less per period.

The original period of the problem is a week and

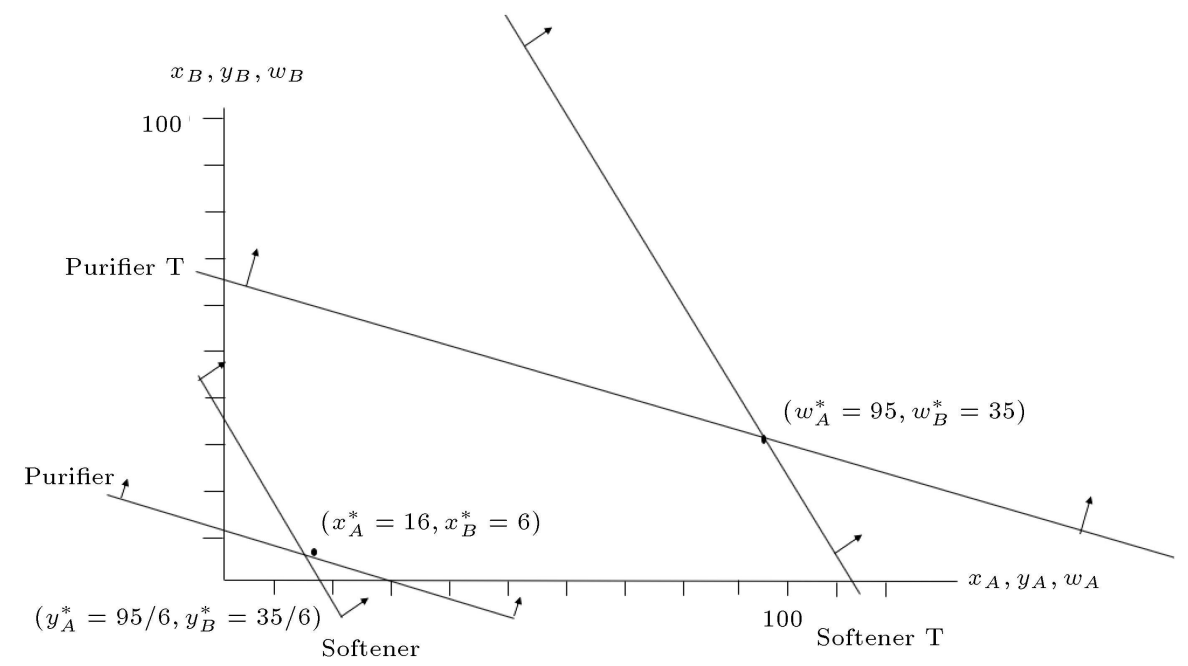

Figure 1. Geometrical representation of the three solution spaces for the illustration in Section 4.1. 
Table 3. Airline fleet capacity and cost per route for the airline case.

\begin{tabular}{ccccccc}
\hline Fleet & Seat capacity & Availability & Route 1 & Route 2 & Route 3 & Route 4 \\
\hline 1 & 200 & 5 & 5 & 4 & 3 & 2 \\
2 & 150 & 3 & 7 & 5 & 5 & 4 \\
3 & 120 & 2 & 9 & 7 & 6 & 6
\end{tabular}

that the manufacturing facility works for an undetermined number of weeks. A practical solution is to manufacture 48 units of product two. At the end of the first 44 hours per week, the 49 -th unit will be finished at $8 / 9 \approx 0.8888(88.88 \%)$. Assuming that the process can stop at no loss of any kind and resume in the next consecutive period, the 49-th unit (needing only $1 / 9$ of the work) at the beginning of the second period is considered to continue the process until finishing it and the manufacturing process continues. In doing so, in eight out of nine weeks, the manufacturing process delivers 49 units of product 2 and only 48 units of product 2 will be delivered in one out of nine weeks. Hence, in 9 weeks, 440 units of product two are delivered, which is an integer number.

In doing so, the average production per week is $440 / 9 \approx 48.88$ units of product two and the average objective function value is $\$ 5,133.33$ per week with all the constraints met. This is possible because an inventory of partially-finished units could be conveyed from one period to the next. Notice that the five characteristics of PCIPLPs are met.

This process can be extended to any number of products and resources in the same modeling.

Notice that the proposed solution is neither the solution to Relation (9) nor the integer version of Relation (9). It satisfies the practitioner's requirements in nine weeks.

\subsection{An airline opening route decision}

An airline company is analyzing the opening of four new routes to be assigned to their newly acquired fleet. Tables 3, 4, and 5 show the related data [20]. The relaxed model is defined as follows:

$$
\begin{aligned}
& \min 250,000 y_{1,1}+280,000 y_{1,2}+120,000 y_{1,3} \\
&+80,000 y_{1,4}+245,000 y_{2,1}+400,000 y_{2,2} \\
&+125,000 y_{2,3}+200,000 y_{2,4}+252,000 y_{3,1}
\end{aligned}
$$

Table 4. Demand and opportunity cost per route for the airline case.

\begin{tabular}{ccc}
\hline Route & $\begin{array}{c}\text { Passenger demand } \\
\text { per day }\end{array}$ & $\begin{array}{c}\text { Opportunity cost } \\
\text { per empty seat }\end{array}$ \\
\hline 1 & 2,500 & 2,500 \\
2 & 2,000 & 3,000 \\
3 & 2,200 & 2,800 \\
4 & 1,800 & 2,950 \\
\hline
\end{tabular}

Table 5. Operational cost per route for the airline case.

\begin{tabular}{ccccc}
\hline Fleet & Route 1 & Route 2 & Route 3 & Route 4 \\
\hline 1 & 50,000 & 70,000 & 40,000 & 40,000 \\
2 & 35,000 & 80,000 & 25,000 & 50,000 \\
3 & 28,000 & 45,000 & 27,000 & 26,000 \\
\hline & $+315,000 y_{3,2}+162,000 y_{3,3}+156,000 y_{3,4}$ \\
& $+2,500$ nfp $_{1}+3,000$ nfp $_{2}+2,800$ nf $_{3}$ \\
& $+2,950$ nfp $_{4}$,
\end{tabular}

s.t.:

Fleet 1

$$
y_{1,1}+y_{1,2}+y_{1,3}+y_{1,4} \leq 5,
$$

Fleet 2

$$
y_{2,1}+y_{2,2}+y_{2,3}+y_{2,4} \leq 3
$$

Fleet 3

$$
y_{3,1}+y_{3,2}+y_{3,3}+y_{3,4} \leq 2,
$$

Seats route 1

$$
\begin{array}{r}
1,000 y_{1,1}+1,050_{y 2,1}+1,080 y_{3,1} \\
+n f p_{1}-e s_{1}=2,500
\end{array}
$$

Seats route $2 \quad 800 y_{1,2}+750 y_{2,2}+840 y_{3,2}$

$$
+n f p_{2}-e s_{2}=2,000
$$

Seats route 3

$$
\begin{aligned}
600 y_{1,3} & +750 y_{2,3}+720 y_{3,3} \\
& +n f p_{3}-e s_{3}=2,200
\end{aligned}
$$

Seats route $4 \quad 400 y_{1,4}+600 y_{2,4}+720 y_{3,4}$

$$
+n f p_{4}-e s_{4}=1,800
$$

$$
y_{i, j}, n f p_{j}, e s_{i} \geq 0, \quad \forall i=1,2,3, \quad \text { and } \quad j=1,2,3,4,
$$

where $y_{i, j}$ represents the number of airplanes from fleet $i$ to be assigned to fly route $j$ per a day; $n f p_{j}$ defines the number of passengers (per day) under demand for route $j$ (passenger not flown); and $e s_{i}$ is equal to the number of empty seats flown in a day for the $j$ th route.

The optimal continuous solution is: $y_{1,1}=5 / 2$, $y_{1,2}=5 / 2, y_{2,3}=44 / 15, y_{2,4}=1 / 15, y_{3,4}=2$, and $n f p_{4}=320$, as seen in Table 6 , which shows the nonzero elements of the solution. The minimum daily cost is $\$ 2,961,000.00$. If the integrality constraint is 
Table 6. Optimal continuous daily solution for the airline case, with cost of $\$ 2,961,000.00$.

\begin{tabular}{ccccc}
\hline Fleet & Route 1 & Route 2 & Route 3 & Route 4 \\
\hline 1 & $5 / 2$ & $5 / 2$ & & \\
2 & & & $44 / 15$ & $1 / 15$ \\
3 & & & & 2 \\
& & & & \\
$n f p$ & & & \\
$e s$ & & & \\
\hline
\end{tabular}

Table 7. Optimal continuous solution for the airline case, with $T=30$, and average daily cost of $\$ 2,961,000.00$.

\begin{tabular}{ccccc}
\hline Fleet & Route $\mathbf{1}$ & Route 2 & Route 3 & Route 4 \\
\hline 1 & 75 & 75 & & \\
2 & & & 88 & 2 \\
3 & & & & 60 \\
& & & & \\
$n f p$ & & & 9,600 \\
$e s$ & & & \\
\hline
\end{tabular}

included, the objective function rises to $\$ 4,259,000.00$, i.e., an approximate increase in the daily cost of $43.84 \%$.

From Table 6, the minimum common multiple of $\{2,15\}$ is $T=30$. Considering that the flight plan contemplates 30 days now, Table 7 shows a solution for that period, maintaining a 30-day cost of $\$ 88,830,000.00$, i.e., an average daily cost at $\$ 2,961,000.00$.

\subsection{Industrial size order acceptance case}

An automotive Original Equipment Manufacturer (OEM) is asked to quote orders (products) for a new automotive platform. The potential contract includes a non-determined large number of periods. The OEM has manufacturing technical capabilities to quote ninety-three orders $(m=93)$. Each order to be quoted is expected to be manufactured on a highly specialized manufacturing cell. For the purpose of this case, the manufacturing cell can be considered a single machine. This manufacturing cell works three shifts of eight hours, each. The manufacturing cell utilization factor is 85 percent, making 36,720 working minutes available per month (available time, $A T=36,720$ ).

Table 8 shows the monthly demand for each order, setup time (in minutes), marginal contribution of each unit in USD, the setup cost in USD, and the manufacturing standard time per unit, respectively. Five main raw materials $(m=5)$ are needed for every unit in each order. The current monthly availability of these raw materials is $R M=\{9,000 ; 8,000 ; 7,000 ; 6,000 ; 3,000\}$. The unitary requirement of each raw material is also shown in Table 8.
The relaxed mathematical formulation of the problem in a general form as follows:

$$
\begin{aligned}
& \max \sum_{i \in I} M C_{i} y_{i}-\sum_{i \in I} S U C_{i} a_{i}, \\
& \text { s.t.: } \\
& \text { Demand: } y_{i} \leq a_{i} d_{i}, \quad \forall i \in I, \\
& \text { Time availability: } \quad \sum_{i \in I}\left(S U T_{i} a_{i}+S T_{i} y_{i}\right) \leq A T, \\
& \text { Row material availability: } \quad \sum_{i \in I} c_{i, j} y_{i} \leq R M_{j}, \\
& a_{i} \in \mathbb{B}, \quad x_{i} \in \mathbb{R}, \quad \forall i \in I, \quad \forall j \in J,
\end{aligned}
$$

where $I=\{1, \cdots, n\}$ is the set of $n$ orders and $J=\{1, \cdots, m\}$ is the set of $m$ raw materials, $d_{i}$, $M C_{i}, S U C_{i}, S U T_{i}, S T_{i}$ represent demand, marginal contribution, setup cost, and setup time for the $i$ th order, respectively; $c_{i, j}$ defines the number of units from raw material $j$ where each product in order $i$ requires, $A T$ states the available manufacturing time per period, and $R M_{j}$ contains the availability of the $j$ th raw material per period. The decision variable $y_{i}$ defines the number of units to accept from the $i$ th order, while $a_{i}$ represents auxiliary variable to model the setups.

If all orders on full demand are accepted, an operational profit (marginal contribution minus setup costs) of $328,436.00$ USD is achieved, but that solution requires 120,449 minutes per month (while only 36,700 are available) from the manufacturing cell, while the raw material requirements for this solution are 29,330 units of raw material one (while 9,000 are available); 28,392 units of raw material two (only 8,000 available); 23,791 units of raw material three (only 7,000 available); 20,021 units of raw material four (only 6,000 available), and finally 15,529 units of raw material five (only 3,000 available).

Since accepting all orders is not feasible, the order acceptance problem consists of maximizing the average monthly operational profit by deciding what orders to accept and what manufacturing level to run (units per month) in the case of accepted orders.

Table 9 shows the optimal relaxed solution (up to two digits after the decimal point), which makes $142,850.34$ USD of operational profit, while the integer solution makes 137,314 USD. Notice that the relaxed solution increases the monthly operational profit by 5,536.34 USD (approximately $4.03 \%$ increase).

In the relaxed solution, orders 20 (14.32 units), 38 (113.26 units), and 43 (44.11 units) have no integer 
Table 8. Industrial size order acceptance problem data.

\begin{tabular}{|c|c|c|c|c|c|c|c|c|c|c|}
\hline \multirow{2}{*}{$\begin{array}{l}\text { Order } \\
\quad(i)\end{array}$} & \multirow{2}{*}{$\begin{array}{l}\text { Demand } \\
\qquad\left(d_{i}\right)\end{array}$} & \multirow{2}{*}{$\begin{array}{l}\text { Set up } \\
\left(S U T_{i}\right)\end{array}$} & \multirow{2}{*}{$\begin{array}{l}\text { Marg. cont. } \\
\qquad\left(M C_{i}\right)\end{array}$} & \multirow{2}{*}{$\begin{array}{l}\text { Set up } \\
\left(S U C_{i}\right)\end{array}$} & \multirow{2}{*}{$\begin{array}{l}\text { Std. time } \\
\qquad\left(S T_{i}\right)\end{array}$} & \multicolumn{5}{|c|}{ Raw materials per unit } \\
\hline & & & & & & $c_{i, 1}$ & $c_{i, 2}$ & $c_{i, 3}$ & $c_{i, 4}$ & $c_{i, 5}$ \\
\hline 1 & 62 & 86 & 68 & 66 & 14 & 1 & 6 & 3 & 3 & 1 \\
\hline 2 & 129 & 63 & 26 & 100 & 14 & 7 & 1 & 3 & 4 & 3 \\
\hline 3 & 114 & 82 & 23 & 84 & 18 & 3 & 4 & 2 & 3 & 2 \\
\hline 4 & 94 & 89 & 15 & 77 & 17 & 4 & 3 & 5 & 1 & 1 \\
\hline 5 & 20 & 52 & 73 & 70 & 11 & 2 & 2 & 1 & 4 & 1 \\
\hline 6 & 27 & 57 & 25 & 50 & 23 & 2 & 4 & 1 & 4 & 2 \\
\hline 7 & 125 & 67 & 70 & 98 & 18 & 1 & 4 & 1 & 4 & 1 \\
\hline 8 & 95 & 82 & 7 & 92 & 20 & 1 & 4 & 3 & 2 & 1 \\
\hline 9 & 74 & 80 & 68 & 95 & 11 & 1 & 4 & 5 & 2 & 3 \\
\hline 10 & 143 & 26 & 29 & 34 & 20 & 2 & 6 & 2 & 4 & 1 \\
\hline 11 & 113 & 61 & 70 & 10 & 7 & 3 & 6 & 2 & 4 & 3 \\
\hline 12 & 20 & 8 & 79 & 96 & 21 & 1 & 1 & 4 & 1 & 3 \\
\hline 13 & 21 & 20 & 59 & 95 & 9 & 4 & 1 & 5 & 3 & 3 \\
\hline 14 & 130 & 45 & 28 & 64 & 23 & 5 & 2 & 1 & 1 & 2 \\
\hline 15 & 114 & 64 & 34 & 91 & 23 & 3 & 6 & 4 & 2 & 1 \\
\hline 16 & 100 & 96 & 25 & 38 & 12 & 6 & 5 & 1 & 2 & 1 \\
\hline 17 & 124 & 87 & 62 & 23 & 9 & 5 & 5 & 2 & 3 & 1 \\
\hline 18 & 136 & 89 & 77 & 10 & 7 & 1 & 2 & 4 & 4 & 3 \\
\hline 19 & 34 & 61 & 50 & 46 & 8 & 4 & 4 & 1 & 2 & 2 \\
\hline 20 & 70 & 99 & 56 & 12 & 8 & 6 & 4 & 3 & 2 & 2 \\
\hline 21 & 148 & 24 & 47 & 82 & 16 & 7 & 4 & 5 & 1 & 2 \\
\hline 22 & 88 & 32 & 82 & 92 & 13 & 6 & 5 & 5 & 3 & 3 \\
\hline 23 & 103 & 56 & 76 & 31 & 7 & 6 & 6 & 2 & 2 & 2 \\
\hline 24 & 132 & 7 & 65 & 69 & 10 & 3 & 6 & 1 & 2 & 3 \\
\hline 25 & 95 & 73 & 6 & 61 & 18 & 3 & 3 & 4 & 4 & 3 \\
\hline 26 & 42 & 45 & 19 & 7 & 19 & 2 & 6 & 2 & 2 & 1 \\
\hline 27 & 130 & 35 & 31 & 51 & 8 & 2 & 2 & 4 & 4 & 3 \\
\hline 28 & 143 & 73 & 29 & 22 & 19 & 2 & 3 & 2 & 4 & 2 \\
\hline 29 & 127 & 25 & 8 & 40 & 8 & 6 & 4 & 5 & 1 & 2 \\
\hline 30 & 33 & 41 & 16 & 7 & 13 & 1 & 5 & 2 & 4 & 3 \\
\hline 31 & 55 & 34 & 21 & 90 & 7 & 3 & 5 & 1 & 3 & 1 \\
\hline 32 & 52 & 94 & 25 & 62 & 9 & 1 & 4 & 1 & 1 & 3 \\
\hline 33 & 19 & 35 & 29 & 19 & 22 & 7 & 6 & 1 & 4 & 3 \\
\hline 34 & 138 & 53 & 27 & 91 & 22 & 4 & 3 & 4 & 4 & 3 \\
\hline 35 & 73 & 49 & 68 & 38 & 19 & 3 & 4 & 1 & 1 & 1 \\
\hline 36 & 76 & 32 & 59 & 89 & 12 & 3 & 1 & 1 & 3 & 2 \\
\hline 37 & 93 & 58 & 42 & 66 & 6 & 3 & 5 & 3 & 2 & 1 \\
\hline 38 & 116 & 57 & 48 & 85 & 22 & 2 & 6 & 2 & 4 & 1 \\
\hline 39 & 127 & 35 & 21 & 44 & 6 & 4 & 1 & 3 & 1 & 2 \\
\hline 40 & 63 & 20 & 67 & 66 & 14 & 5 & 1 & 3 & 4 & 1 \\
\hline 41 & 142 & 50 & 22 & 59 & 12 & 4 & 6 & 2 & 1 & 3 \\
\hline 42 & 96 & 58 & 13 & 78 & 18 & 5 & 6 & 4 & 1 & 1 \\
\hline 43 & 128 & 53 & 54 & 62 & 21 & 7 & 4 & 5 & 3 & 1 \\
\hline
\end{tabular}


Table 8. Industrial size order acceptance problem data (continued).

\begin{tabular}{|c|c|c|c|c|c|c|c|c|c|c|}
\hline \multirow{2}{*}{$\begin{array}{c}\text { Order } \\
(i)\end{array}$} & \multirow{2}{*}{$\begin{array}{c}\text { Demand } \\
\left(d_{i}\right)\end{array}$} & \multirow{2}{*}{$\begin{array}{l}\text { Set up } \\
\left(S U T_{i}\right) \\
\end{array}$} & \multirow{2}{*}{$\begin{array}{l}\text { Marg. cont. } \\
\quad\left(M C_{i}\right)\end{array}$} & \multirow{2}{*}{$\begin{array}{l}\text { Set up } \\
\left(S U C_{i}\right)\end{array}$} & \multirow{2}{*}{$\begin{array}{l}\text { Std. time } \\
\quad\left(S T_{i}\right)\end{array}$} & \multicolumn{5}{|c|}{ Raw materials per unit } \\
\hline & & & & & & $c_{i, 1}$ & $c_{i, 2}$ & $c_{i, 3}$ & $c_{i, 4}$ & $c_{i, 5}$ \\
\hline 44 & 78 & 69 & 66 & 65 & 15 & 4 & 1 & 5 & 1 & 3 \\
\hline 45 & 50 & 73 & 13 & 11 & 25 & 7 & 1 & 2 & 1 & 1 \\
\hline 46 & 69 & 91 & 78 & 71 & 18 & 3 & 1 & 4 & 1 & 3 \\
\hline 47 & 123 & 24 & 27 & 29 & 6 & 6 & 5 & 2 & 4 & 3 \\
\hline 48 & 39 & 44 & 59 & 10 & 6 & 3 & 6 & 3 & 2 & 3 \\
\hline 49 & 45 & 11 & 62 & 32 & 16 & 7 & 3 & 5 & 2 & 1 \\
\hline 50 & 67 & 31 & 41 & 91 & 8 & 4 & 2 & 2 & 4 & 2 \\
\hline 51 & 50 & 29 & 10 & 68 & 18 & 7 & 3 & 5 & 1 & 2 \\
\hline 52 & 23 & 74 & 30 & 38 & 11 & 3 & 3 & 5 & 1 & 1 \\
\hline 53 & 128 & 11 & 37 & 58 & 18 & 3 & 5 & 3 & 1 & 2 \\
\hline 54 & 113 & 68 & 70 & 82 & 16 & 4 & 4 & 5 & 3 & 3 \\
\hline 55 & 57 & 49 & 43 & 26 & 13 & 7 & 2 & 4 & 3 & 2 \\
\hline 56 & 125 & 67 & 64 & 57 & 6 & 3 & 1 & 5 & 4 & 1 \\
\hline 57 & 103 & 86 & 60 & 42 & 8 & 2 & 1 & 5 & 1 & 3 \\
\hline 58 & 76 & 50 & 80 & 16 & 13 & 4 & 5 & 2 & 3 & 2 \\
\hline 59 & 121 & 78 & 74 & 35 & 11 & 4 & 6 & 4 & 4 & 2 \\
\hline 60 & 137 & 33 & 52 & 26 & 16 & 6 & 2 & 4 & 1 & 1 \\
\hline 61 & 118 & 71 & 45 & 77 & 7 & 3 & 6 & 3 & 4 & 1 \\
\hline 62 & 74 & 19 & 16 & 83 & 23 & 2 & 4 & 3 & 2 & 1 \\
\hline 63 & 143 & 56 & 68 & 59 & 14 & 2 & 6 & 4 & 2 & 2 \\
\hline 64 & 22 & 24 & 63 & 94 & 24 & 4 & 5 & 3 & 3 & 2 \\
\hline 65 & 54 & 18 & 37 & 67 & 10 & 3 & 3 & 3 & 4 & 3 \\
\hline 66 & 47 & 99 & 28 & 12 & 15 & 1 & 2 & 1 & 3 & 3 \\
\hline 67 & 148 & 98 & 15 & 92 & 24 & 7 & 6 & 1 & 1 & 3 \\
\hline 68 & 20 & 33 & 55 & 19 & 14 & 1 & 4 & 3 & 1 & 1 \\
\hline 69 & 54 & 27 & 8 & 76 & 7 & 1 & 3 & 5 & 2 & 2 \\
\hline 70 & 114 & 77 & 81 & 26 & 24 & 4 & 1 & 5 & 1 & 1 \\
\hline 71 & 62 & 42 & 11 & 60 & 9 & 4 & 1 & 3 & 2 & 2 \\
\hline 72 & 43 & 64 & 20 & 47 & 21 & 1 & 6 & 5 & 1 & 2 \\
\hline 73 & 67 & 14 & 19 & 41 & 16 & 3 & 4 & 2 & 1 & 1 \\
\hline 74 & 53 & 82 & 34 & 52 & 19 & 4 & 5 & 3 & 3 & 3 \\
\hline 75 & 141 & 33 & 71 & 44 & 22 & 1 & 3 & 2 & 3 & 3 \\
\hline 76 & 81 & 60 & 14 & 83 & 23 & 4 & 6 & 2 & 3 & 2 \\
\hline 77 & 19 & 70 & 23 & 69 & 8 & 2 & 2 & 4 & 3 & 3 \\
\hline 78 & 141 & 31 & 73 & 61 & 14 & 6 & 1 & 4 & 4 & 2 \\
\hline 79 & 18 & 69 & 59 & 50 & 22 & 6 & 1 & 4 & 1 & 3 \\
\hline 80 & 119 & 77 & 15 & 76 & 18 & 3 & 2 & 1 & 1 & 1 \\
\hline 81 & 19 & 23 & 77 & 94 & 16 & 5 & 3 & 1 & 1 & 1 \\
\hline 82 & 23 & 59 & 53 & 51 & 21 & 4 & 1 & 5 & 3 & 2 \\
\hline 83 & 42 & 80 & 49 & 26 & 24 & 5 & 4 & 4 & 2 & 3 \\
\hline 84 & 146 & 45 & 83 & 81 & 23 & 5 & 2 & 5 & 4 & 1 \\
\hline 85 & 47 & 82 & 38 & 65 & 19 & 4 & 1 & 3 & 3 & 3 \\
\hline 86 & 112 & 60 & 8 & 94 & 7 & 1 & 2 & 2 & 4 & 1 \\
\hline
\end{tabular}


Table 8. Industrial size order acceptance problem data (continued).

\begin{tabular}{|c|c|c|c|c|c|c|c|c|c|c|}
\hline \multirow{2}{*}{$\begin{array}{l}\text { Order } \\
\quad(i)\end{array}$} & \multirow{2}{*}{$\begin{array}{l}\text { Demand } \\
\qquad\left(d_{i}\right)\end{array}$} & \multirow{2}{*}{$\begin{array}{l}\text { Set up } \\
\left(S U T_{i}\right)\end{array}$} & \multirow{2}{*}{$\begin{array}{l}\text { Marg. cont. } \\
\qquad\left(M C_{i}\right)\end{array}$} & \multirow{2}{*}{$\begin{array}{l}\text { Set up } \\
\left(S U C_{i}\right)\end{array}$} & \multirow{2}{*}{$\begin{array}{c}\text { Std. time } \\
\quad\left(S T_{i}\right)\end{array}$} & \multicolumn{5}{|c|}{ Raw materials per unit } \\
\hline & & & & & & $c_{i, 1}$ & $c_{i, 2}$ & $c_{i, 3}$ & $c_{i, 4}$ & $c_{i, 5}$ \\
\hline 87 & 46 & 7 & 76 & 32 & 23 & 7 & 3 & 3 & 2 & 2 \\
\hline 88 & 149 & 84 & 14 & 28 & 6 & 7 & 2 & 2 & 3 & 3 \\
\hline 89 & 29 & 18 & 80 & 50 & 10 & 1 & 1 & 5 & 4 & 1 \\
\hline 90 & 67 & 41 & 8 & 20 & 14 & 1 & 2 & 1 & 1 & 3 \\
\hline 91 & 36 & 24 & 49 & 43 & 19 & 3 & 3 & 3 & 4 & 2 \\
\hline 92 & 114 & 60 & 9 & 80 & 24 & 2 & 4 & 4 & 4 & 2 \\
\hline 93 & 83 & 58 & 28 & 43 & 10 & 2 & 6 & 2 & 1 & 2 \\
\hline
\end{tabular}

Table 9. Relaxed versus integer solutions.

\begin{tabular}{|c|c|c|c|}
\hline $\begin{array}{c}\text { Op. }{ }^{a} \\
\text { profit }\end{array}$ & & $\$ 142,850.34$ & $\$ 137,314.00$ \\
\hline Order & Demand $(u)$ & Relaxed & Integer \\
\hline 1 & 62 & 62 & 62 \\
\hline 5 & 20 & 20 & 20 \\
\hline 7 & 125 & 125 & 100 \\
\hline 15 & 114 & 0 & 63 \\
\hline 17 & 124 & 124 & 100 \\
\hline 18 & 136 & 0 & 10 \\
\hline 20 & 70 & 14.32 & 70 \\
\hline 23 & 103 & 103 & 100 \\
\hline 35 & 73 & 73 & 73 \\
\hline 36 & 76 & 76 & 75 \\
\hline 37 & 93 & 93 & 93 \\
\hline 38 & 116 & 113.26 & 100 \\
\hline 40 & 63 & 63 & 63 \\
\hline 43 & 128 & 44.11 & 100 \\
\hline 46 & 69 & 69 & 69 \\
\hline 49 & 45 & 45 & 45 \\
\hline 56 & 125 & 125 & 100 \\
\hline 58 & 76 & 76 & 76 \\
\hline 59 & 121 & 121 & 100 \\
\hline 60 & 137 & 137 & 100 \\
\hline 61 & 118 & 0 & 98 \\
\hline 63 & 143 & 143 & 100 \\
\hline 64 & 22 & 0 & 22 \\
\hline 68 & 20 & 20 & 20 \\
\hline 70 & 114 & 114 & 100 \\
\hline 78 & 141 & 141 & 100 \\
\hline 81 & 19 & 19 & 19 \\
\hline 84 & 146 & 146 & 100 \\
\hline 87 & 46 & 46 & 46 \\
\hline 89 & 29 & 29 & 29 \\
\hline
\end{tabular}

a: Op.: Operational

production units. Therefore, order 20 will deliver 15 units in $32 \%$ of the months while 14 units in the rest; order 38 will deliver 114 units in $26 \%$ of the months and 113 in the rest; and finally, order 43 will deliver 45 units in $11 \%$ of the months and 44 in the rest.

For a literature review of order acceptance, please refer to [21].

\section{Conclusions and further research}

Many real-world problems require formulating and solving an integer programming model. The integer solution generally produces a significant worsened objective function value compared with the objective function value of the corresponding relaxed linear programming model. Thus, it is important to explore the possibility of generating a feasible integer solution that maintains the objective value of the relaxed problem at least on average per decision period.

This study shows a class of integer programming problems named Pseudo-Continuous-Integer Periodical Linear Problem (PCIPLP) in which the relaxed solution is used as the basis to construct a feasible integer solution maintaining the value of the relaxed solution by proposing a change in the problem planning horizon. One significant fact of our approach is that the provided integer solution is not an optimal solution for neither the original integer model proposed nor its relaxed version given the changes in the problem planning horizon made. The provided integer solution can be implemented in practice and maintains the objective function value of the relaxed problem on average (per period).

The set of PCIPLPs consists of integer single period problems that repeats indefinitely, but elements from one period can be conveyed to the next, thus a change in the problem planning horizon from one period to $T$ periods is feasible, where $T$ is computed as the minimum common multiple of the optimum continuous decision variable denominator values.

Further research pends ahead:

(a) Identification of some other kinds of integer problems that can be treated in a similar way; 
(b) Identification of a set of mix-integer problems with similar characteristics;

(c) Search for classes of non-linear integer (or mixinteger) programming problems that can be solved in the same manner;

(d) Exploration of the effectiveness of this methodology for solving large-scale problems;

(e) Since sensitivity analysis can be applied to Relations (3) to (5) and its results are practical as long as the resultant solution remains fully integer, new techniques and new characteristics must be developed and found, respectively, to perform sensitivity analysis on a more practical matter.

\section{References}

1. Oluleye, G., Vasquez, L., Smith, R., et al. "A multiperiod mixed integer linear program for design of residential distributed energy centres with thermal demand data discretisatio", Sustainable Production and Consumption, 5, pp. 16-28 (2016).

2. Tan, R.R. "A multi-period source-sink mixed integer linear programming model for biochar-based carbon sequestration systems", Sustainable Production and Consumption, 8, pp. 57-63 (2016).

3. Ciavotta, M., Ardagna, D., and Gibilisco, G.P. "A mixed integer linear programming optimization approach for multi-cloud capacity allocation", Journal of Systems and Software, 123, pp. 64-78 (2017).

4. Oluleye, G., Allison, J., Kelly, N., et al. "A multiperiod mixed integer linear program for assessing the benefits of power to heat storage in a dwelling energy system", In Computer Aided Chemical Engineering, 43, pp. 1451-1456 (2018).

5. Zeng, Y., Xiao, X., Li, J., et al. "A novel multi-period mixed-integer linear optimization model for optimal distribution of byproduct gases, steam and power in an iron and steel plant", Energy, 143, pp. 881-899 (2018).

6. Bastos, L.S., Marchesi, J.F., Hamacher, S., et al. "A mixed integer programming approach to the patient admission scheduling problem", European Journal of Operational Research, 273(3), pp. 831-840 (2019).

7. Dorneanu, B., Sidnell, T., Clarke, F., et al. "A Mixedinteger linear programming model for the optimal operation and design of residential neighbourhoods", IFAC-Papers OnLine, 52(1), pp. 934-939 (2019).

8. Abbaszadeh, N., Asadi-Gangraj, E., and Emami, S. "Flexible flow shop scheduling problem to minimize makespan with renewable resources", Scientia Iranica, 28(3), pp. 1853-1870 (2021).

9. Abrishami, S.J., Vahdani, H., and Rezaee, B. "An integrated lot-sizing model with supplier and carrier selection and quantity discounts considering multiple products", Scientia Iranica, 27(4), pp. 2140-2156 (2020).
10. Zeballos, L.J., Méndez, C.A., and Povoa, A.P.B. "Mixed-integer linear programming approach for product design for life-cycle profit", Computers \& Industrial Engineering, 137, p. 106079 (2019).

11. Dantzig, G., Linear Programming and Extensions, Princeton University Press (1963).

12. Khachiyan, L.G. "Polynomial algorithms in linear programming", USSR Computational Mathematics and Mathematical Physics, 20(1), pp. 53-72 (1980).

13. Karmarkar, N. "A new polynomial-time algorithm for linear programming", In Proceedings of the Sixteenth Annual ACM Symposium on Theory of Computing, pp. 302-311 (1984).

14. Lawler, E.L. and Wood, D.E. "Branch-and-bound methods: A survey", Operations Research, 14(4), pp. 699-719 (1966).

15. Balas, E. "Intersection cuts-A new type of cutting planes for integer programming", Operations Research, 19(1), pp. 19-39 (1971).

16. Chen, D.-S., Batson, R.G., and Dang, Y. "Branch-andcut approach", Applied Integer Programming: Modeling and Solution, John Wiley \& Sons, pp. 305-333 (2009).

17. Chvatal, V., Linear Programming, Macmillan (1983).

18. Trigos, F. and López, E.M. "Maximising profit for multiple-product, Single-period, single-machine manufacturing under sequential set-up constraints that depend on lot size", International Journal of Production Research, 54(4), pp. 1134-1151 (2016).

19. Applegate, D.L., Cook, W., Dash, S., et al. "Exact solutions to linear programming problems", Operations Research Letters, 35(6), pp. 693-699 (2007).

20. Arreola, J. and Arreola, A., Programación Lineal, una Introducción a la Toma de Decisiones Cuantitativa Thomson, (2003).

21. Slotnick, S.A. "Order acceptance and scheduling: A taxonomy and review", European Journal of Operational Research, 212(1), pp. 1-11 (2011).

\section{Biographies}

Federico Trigos holds a $\mathrm{PhD}$ in Industrial Engineering with dissertation on large-scale optimization (Georgia Institute of Technology, USA), a Master of Finance (EGADE Business School, Tecnológico de Monterrey, México), a Master of Science in Industrial Engineering with major in Production Distribution and Material Handling (Georgia Institute of Technology, USA), a Master in Engineering with major in Operations Research (Tecnológico de Monterrey Campus Monterrey, México), and a Bachelor of Industrial and Systems Engineering (Tecnológico de Monterrey Campus Toluca, México). Dr. Federico Trigos has lectured in several professional and research congresses in the United States, Europe, Asia, and Latin America. 
He has been a visiting professor at the Southern Illinois University at Edwardsville and the Iowa State University, both in the USA. He has been a research visiting scholar at the University of Bristol and the University of Southampton, both in the U.K. He has also been recognized by the Massachusetts Institute of Technology (USA) as an International Faculty Fellow. He held the presidency of the Mexican Institute of Industrial and Systems Engineering (based in Monterrey N.L., México) in 2015. Dr. Trigos is an active researcher publishing and leading at EGADE Business School areas related to the sustainable development of quantitative tools to optimize strategic organizational performance. His research interests include industrial and business statistics, simulation, mathematical programming, engineering economics, corporate and family business finance, and financial investments. He is currently a member of the Mexican National
Researchers System (SNI) and the Mexican Academy of Science (AMC). He is an active member of research groups both in Mexico and the United Kingdom.

Leopoldo Eduardo Cárdenas-Barrón is currently a Professor at the School of Engineering and Sciences at Tecnológico de Monterrey, Campus Monterrey, México. $\mathrm{He}$ is also a faculty member at the Department of Industrial and Systems Engineering at Tecnológico de Monterrey. He was the Associate Director of the Industrial and Systems Engineering programme from 1999 to 2005. Moreover, he was also the Associate Director of the Department of Industrial and Systems Engineering from 2005 to 2009. His research areas primarily include inventory planning and control, logistics, and supply chain. He has published papers and technical notes in several international journals. He has co-authored one book in the field of simulation in Spanish. 